\title{
Computed tomography features of COVID-19 in children
}

\section{A systematic review and meta-analysis}

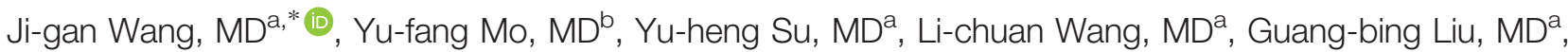 \\ Meng Li, MDa, Qian-qiu Qin, MDa
}

\begin{abstract}
Background: There are few reports on the chest computed tomography (CT) imaging features of children with coronavirus disease 2019 (COVID-19), and most reports involve small sample sizes.

Objectives: To systematically analyze the chest CT imaging features of children with COVID-19 and provide references for clinical practice.

Data sources: We searched PubMed, Web of Science, and Embase; data published by Johns Hopkins University; and Chinese databases CNKI, Wanfang, and Chongqing Weipu.

Methods: Reports on chest CT imaging features of children with COVID-19 from January 1, 2020 to August 10, 2020, were analyzed retrospectively and a meta-analysis carried out using Stata12.0 software.

Results: Thirty-seven articles (1747 children) were included in this study. The heterogeneity of meta-analysis results ranged from $0 \%$ to 90.5\%. The overall rate of abnormal lung CT findings was 63.2\% (95\% confidence interval [Cl]: 55.8\%-70.6\%), with a rate of 61.0\% (95\% Cl: $50.8 \%-71.2 \%)$ in China and $67.8 \%$ (95\% Cl: $57.1 \%-78.4 \%)$ in the rest of the world in the subgroup analysis. The incidence of groundglass opacities was 39.5\% (95\% Cl: 30.7\%-48.3\%), multiple lung lobe lesions was 65.1\% (95\% Cl: 55.1\%-67.9\%), and bilateral lung lesions was 61.5\% (95\% Cl: 58.8\%-72.2\%). Other imaging features included nodules (25.7\%), patchy shadows (36.8\%), halo sign (24.8\%), consolidation (24.1\%), air bronchogram signs (11.2\%), cord-like shadows (9.7\%), crazy-paving pattern (6.1\%), and pleural effusion (9.1\%). Two articles reported 3 cases of white lung, another reported 2 cases of pneumothorax, and another 1 case of bullae.

Conclusions: The lung CT results of children with COVID-19 are usually normal or slightly atypical. The lung lesions of COVID-19 pediatric patients mostly involve both lungs or multiple lobes, and the common manifestations are patchy shadows, ground-glass opacities, consolidation, partial air bronchogram signs, nodules, and halo signs; white lung, pleural effusion, and paving stone signs are rare. Therefore, chest CT has limited value as a screening tool for children with COVID-19 and can only be used as an auxiliary assessment tool.
\end{abstract}

Abbreviations: ACE2 = angiotensin-converting enzyme 2, Cl= confidence interval, COVID-19= coronavirus disease $2019, \mathrm{CT}=$ computed tomography, SARS-CoV-2 = severe acute respiratory syndrome coronavirus 2.

Keywords: children, computed tomography features, COVID-19, SARS-CoV-2

Editor: Vasile Valeriu Lupu.

This work was supported by Self-Funded Scientific Research Project of Guangxi Zhuang Autonomous Region Health Commission (Nos. Z20210123).

The authors declare no conflict of interest.

Supplemental Digital Content is available for this article.

The datasets generated during and/or analyzed during the current study are available from the corresponding author on reasonable request.

a Maternal and Child Health Hospital of Guangxi Zhuang Autonomous Region, Nanning, China, ' Liuzhou Workers' Hospital, Liuzhou, China.

*Correspondence: Ji-gan Wang, 59 Xiangzhu Avenue, Xingning District, Nanning 530003, Guangxi Zhuang Autonomous Region, China

(e-mail: wangjigan@163.com).

Copyright () 2021 the Author(s). Published by Wolters Kluwer Health, Inc This is an open access article distributed under the terms of the Creative Commons Attribution-Non Commercial License 4.0 (CCBY-NC), where it is permissible to download, share, remix, transform, and buildup the work provided it is properly cited. The work cannot be used commercially without permission from the journal.

How to cite this article: Wang Jg, Mo Yf, Su Yh, Wang Lc, Liu Gb, Li M, Qin Qq. Computed tomography features of COVID-19 in children: a systematic review and meta-analysis. Medicine 2021;100:38(e22571).

Received: 10 September 2020 / Received in final form: 30 May 2021 /

Accepted: 6 September 2021

http://dx.doi.org/10.1097/MD.0000000000022571

\section{Introduction}

In January 2020, severe acute respiratory syndrome coronavirus 2 (SARS-CoV-2) was identified as the cause of a series of pneumonia cases first diagnosed in Wuhan, Hubei Province, China. ${ }^{[1]}$ Soon after, SARS-CoV-2 spread all over the world. ${ }^{[2]}$ By March 2020, the spread of coronavirus disease 2019 (COVID-19) was recognized as a pandemic by the World Health Organization. ${ }^{[3]}$ In the early stages of the pandemic, it was thought that children were not easily infected ${ }^{[1]}$; however, as the pandemic has progressed, the number of pediatric cases has gradually increased. Many infected children are asymptomatic, but some patients have fever, dry cough, and fatigue, while others have gastrointestinal symptoms, including abdominal discomfort, nausea, vomiting, abdominal pain, and diarrhea. ${ }^{[4]}$ Computed tomography (CT) is a sensitive tool for diagnosing symptomatic COVID-19 patients. In adult patients, the most common CT manifestation is ground-glass opacities. Other CT manifestations, such as air bronchography, lymph node enlargement, and effusion, are less common. ${ }^{[5]}$ Among the 1014 hospitalized patients with obvious symptoms from Wuhan, China, the CT scans of most patients were abnormal; however, with a sensitivity of $97 \%$ and specificity of $25 \%$, the 
false positive rate was very high. ${ }^{[6]}$ Compared with adults, children have relatively mild symptoms, so CT is not very typical. ${ }^{[7]}$ However, there are few reports on pediatric CT features, and most reports involve small sample sizes. In addition, larger pediatric cohort studies have not been comprehensive enough. ${ }^{[8]}$ Therefore, the lung CT features of children with COVID-19 has been reviewed systematically in this study.

\section{Evidence acquisition}

\subsection{Registration}

This systematic review and meta-analysis was registered in the Prospero International Prospective Register of Systemic Reviews (CRD42020196602).

\subsection{Literature search strategy}

A literature search was conducted through PubMed, Web of Science, Embase, Johns Hopkins University published data, as well as the Chinese databases CNKI, Wanfang, and Chongqing Weipu between January 1, 2020 and August 10, 2020 to collect reports on the characteristics of chest CT of children with COVID-19. Concurrently, online database and manual retrieval were used, and the references included in the literature were traced. Subjectspecific and free words were used in the retrieval, and adjustments were made according to the characteristics of the different databases without limitations to language, race, or region. The following search terms were used: "children," "child," "kid," "pediatric" in association with "clinical feature" OR "epidemiology" OR "Imaging” OR "CT" and "2019-nCoV" OR "COVID19” OR “SARS-CoV-2” OR “Corona Virus Disease 2019.”
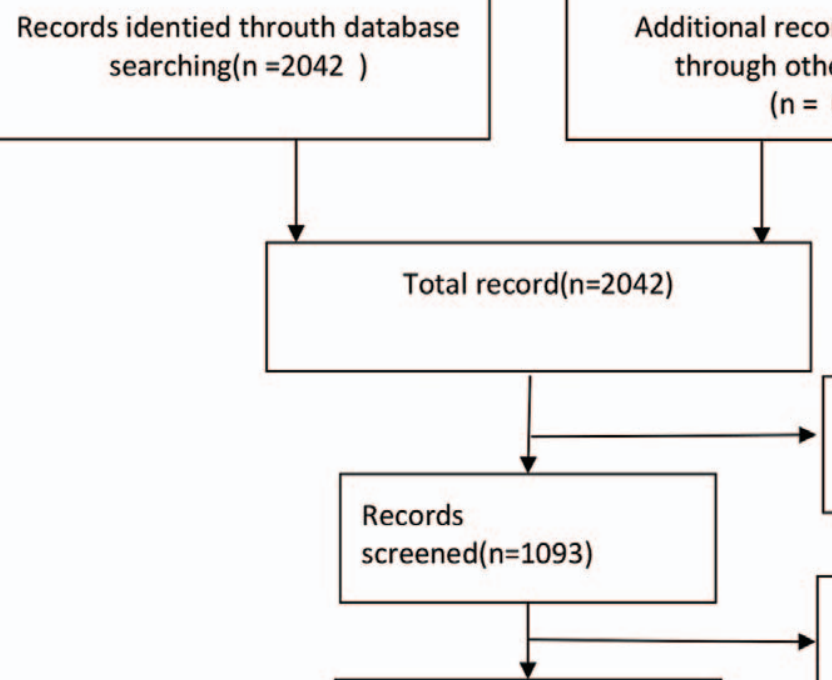

Full-text articles assessed for eligibility ( $n=242)$

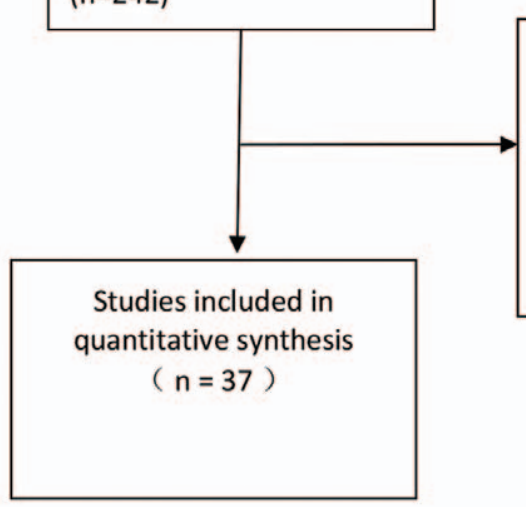

Systematic review $(\mathrm{n}=10)$ Adult studies( $\mathrm{n}=12)$ overlapping or duplicate studies $(\mathrm{n}=50)$

Case reports $(n=45)$

Lacking necessary data (88)

Figure 1. The flowchart of study selection. 


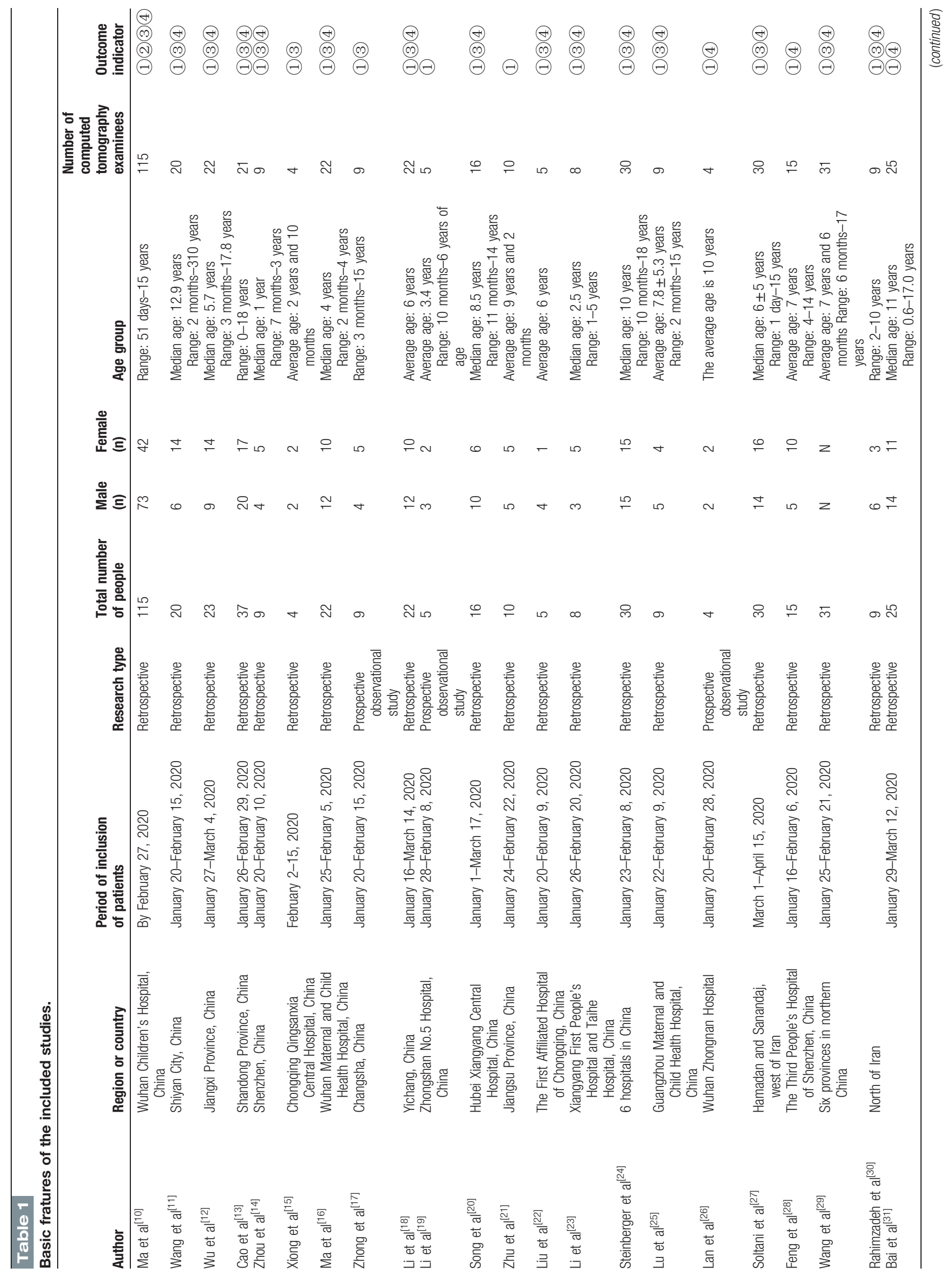




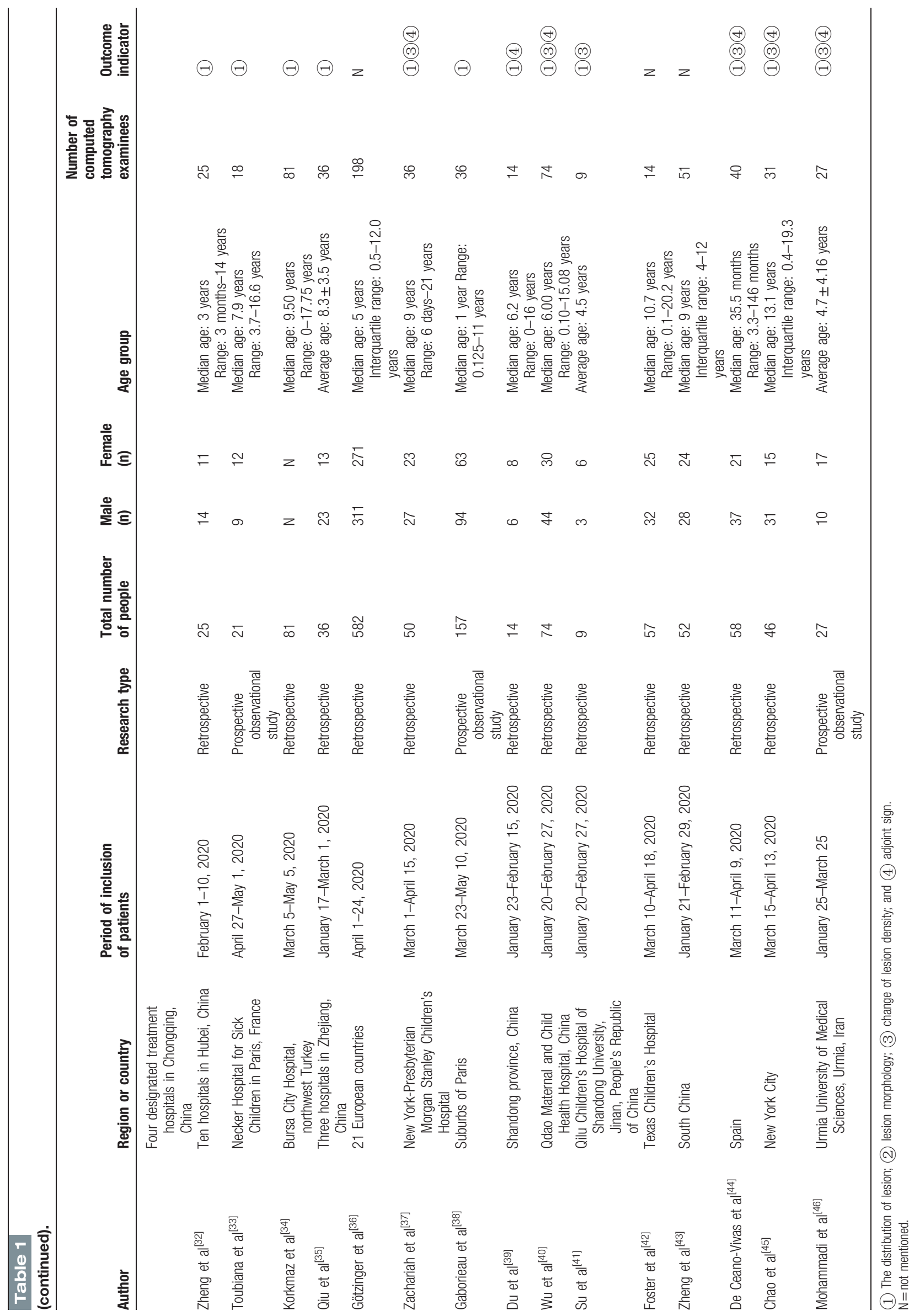


Table 2

Summary of the meta-analysis results on CT image features.

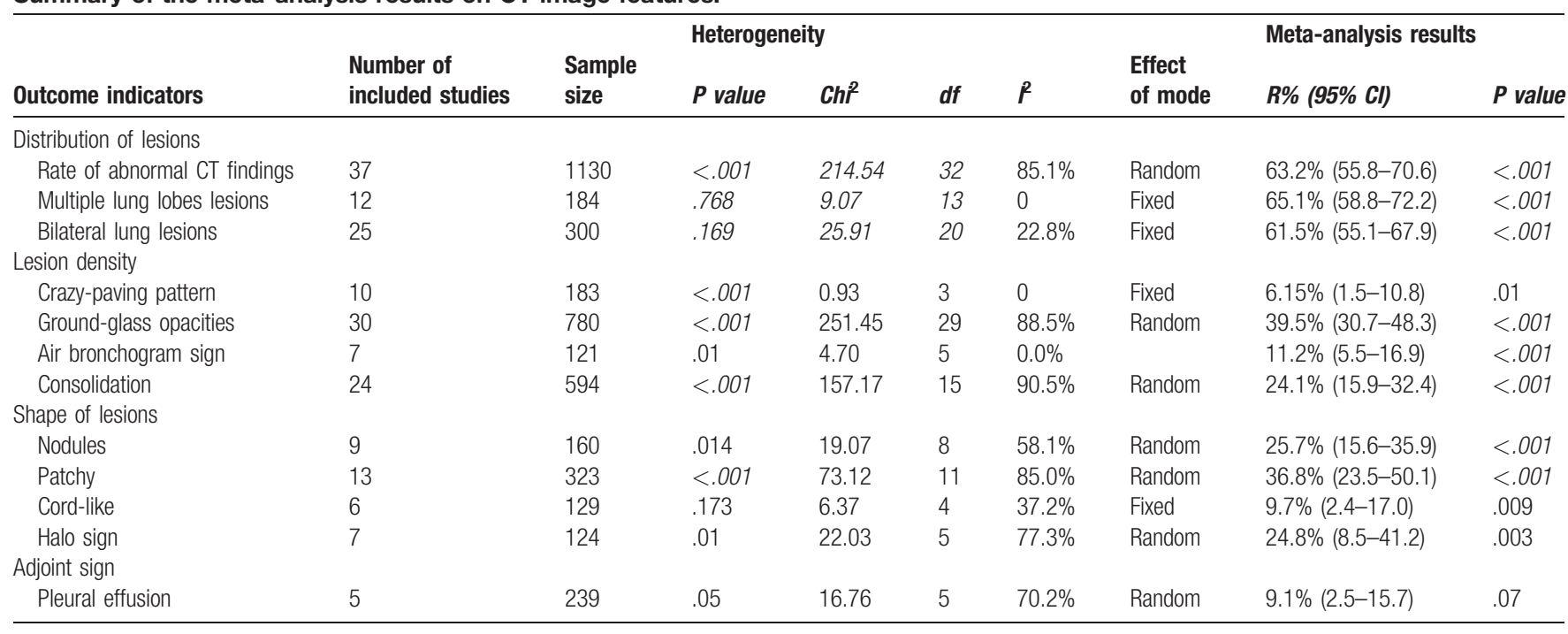

$\mathrm{Cl}=$ confidence interval, $\mathrm{CT}=$ computed tomography.

\subsection{Literature screening and data extraction}

Two researchers independently searched and screened the articles and collected and cross-checked the data. If there was any dispute, it was resolved by a third researcher.

The inclusion criteria consisted of the following: research types: cohort study, case-control study, cross sectional studies, and case analysis; subjects: children with COVID-19; and observation index: imaging features of lung CT or high resolution CT, including lesion distribution, shape, and density change; and accompanying signs.

The exclusion criteria consisted of the following: repeated publications of the same research; short case reports; and incomplete or missing data analysis, without free or easy access to the data.

\subsection{Quality evaluation of the included studies}

This was a case series study that adhered to the National institute for Clinical excellence guidelines for quality evaluation. ${ }^{[9]}$ The evaluation items were as follows: cases in the case series came from medical institutions at different levels and from various difference research centers; the research hypothesis or purpose was clearly described; clear reports were included in the exclusion criteria; measurement results were clearly defined; the collected data achieved the expected purpose; the patient recruitment period was clearly defined; the main findings were clearly described; and results were analyzed and reported in layers. One point was awarded for each item (maximum 8 points), with a total score $\geq 4$ indicating high-quality research. Two researchers independently evaluated the quality and cross-checked the results.

\subsection{Statistical analysis}

A meta-analysis was performed using Stata12.0 software (STATA Corp, College Station, TX). First, the original ratio (R) was transformed by double arcsine to conform to a normal distribution, and then the transformed ratio (TR) was analyzed by meta-analysis. The final rate (r) and its $95 \%$ confidence interval (CI) were finally obtained by converting the results using the formula: $\mathrm{R}=(\sin (\operatorname{tr} / 2))^{2}$. The meta-analysis was carried out using a random-effect model for all studies. The existence of publication bias was judged using the funnel chart, and the significance level was set as $\alpha=0.05$.

\subsection{Ethical statement}

This study was carried out in accordance with the recommendations and in the preferred reporting items for systematic reviews and meta-analyses guidelines. Hence, permission from the ethics committee or the institutional review board is not required.

\section{Evidence synthesis}

\subsection{Literature screening process and results}

A total of 2042 related articles were obtained, which were screened layer by layer. Ultimately, 37 studies were included in this report, ${ }^{[10-46]}$ including 1747 children with COVID-19. Figure 1 shows the process and results of the literature screening.

\subsection{Basic characteristics and quality evaluation results of the included studies}

A total of 37 studies $^{[10-46]}$ were included (11 studies from outside China and 26 studies from China). The included studies were published from January 1, 2020 to August 10, 2020 (Table 1 summarizes the basic characteristics of the included studies; see Table S1, Supplemental Digital Content, http://links.lww.com/ MD2/A466 for details). The quality scores of the included studies ranged from 4 to 8 points, indicating that all were high-quality studies ( $\geq 4$ points, Table S2, Supplemental Digital Content, http://links.lww.com/MD2/A467). 


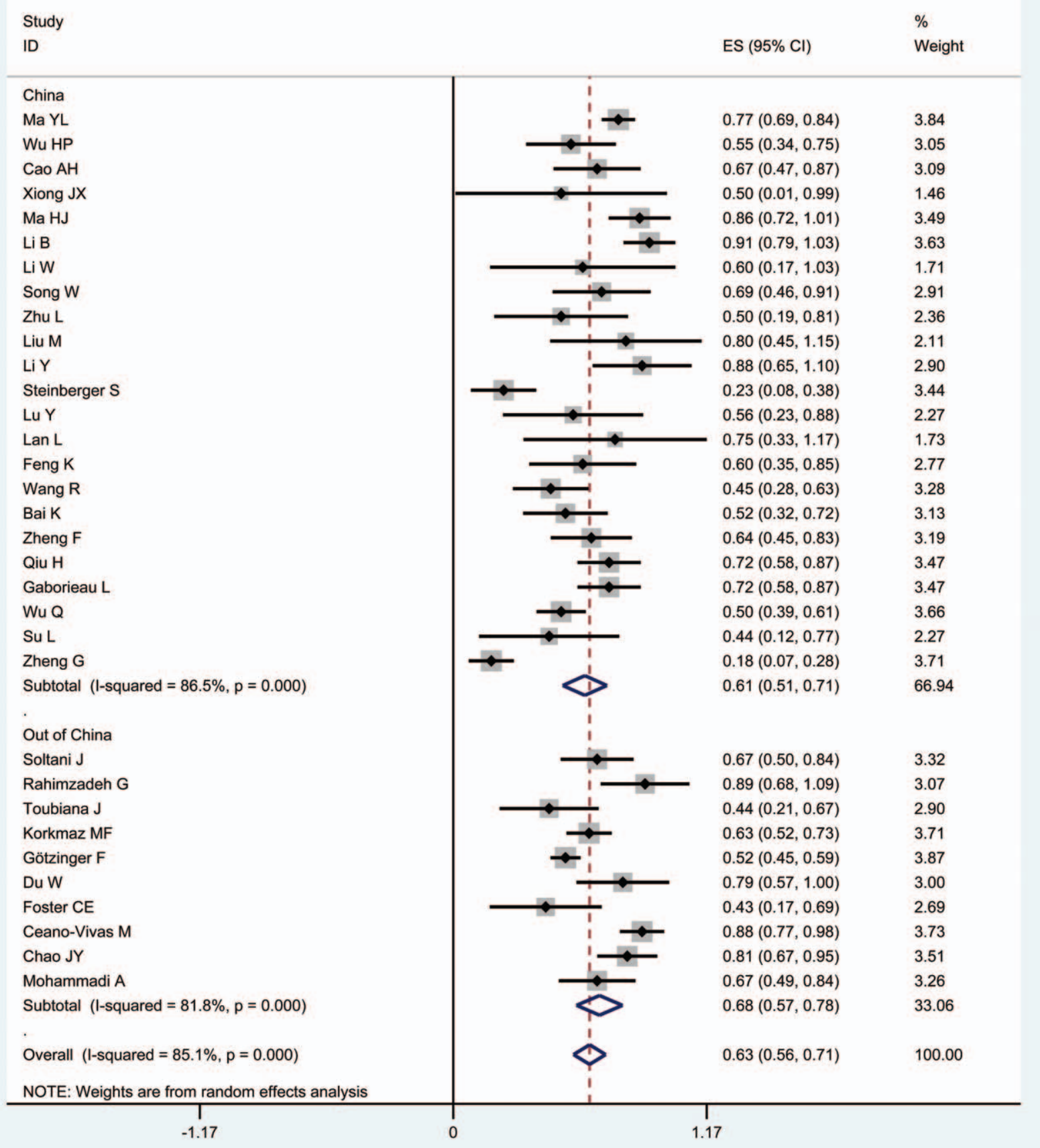

Figure 2. Forest plot of the abnormal pulmonary $\mathrm{CT}$ findings. $\mathrm{Cl}=$ confidence interval, $\mathrm{CT}=$ computed tomography.

\subsection{Meta-analysis results (Table 2)}

3.3.1. Distribution of lesions. Meta-analysis of the randomeffect model showed that the detection rate of abnormal lung CT findings was $63.2 \%$ (95\% CI: $55.8 \%-70.6 \%)$, bilateral lung lesions was $61.5 \%$ (95\% CI: $58.8 \%-72.2 \%)$, and multiple lung lobes lesions was $65.1 \%$ (95\% CI: $55.1 \%-67.9 \%$ ).

3.3.2. Lesion density. Meta-analysis of the random-effect model showed that the prevalence of nodules was $25.7 \%$ (95\% CI: $15.6 \%-35.9 \%)$, patchy shadows was $36.8 \%(95 \%$
CI: $23.5 \%-50.1 \%)$, cord-like pattern was $9.7 \%(95 \%$ CI: $2.4 \%-17 \%)$, and halo signs was $24.8 \%$ (95\% CI: $8.5 \%-$ $10.8 \%)$.

3.3.3. Lesion shape. Meta-analysis of the random-effect model showed that the prevalence of a crazy-paving pattern was $6.15 \%$ (95\% CI: $1.5 \%-10.8 \%$ ), ground-glass opacities was $39.5 \%$ (95\% CI: $30.7 \%-48.3 \%$ ), air bronchogram signs was $11.2 \%$ (95\% CI: $5.5 \%-16.98 \%)$, and consolidation was $24.1 \%(95 \%$ CI: $15.9 \%-41.2 \%$ ). 


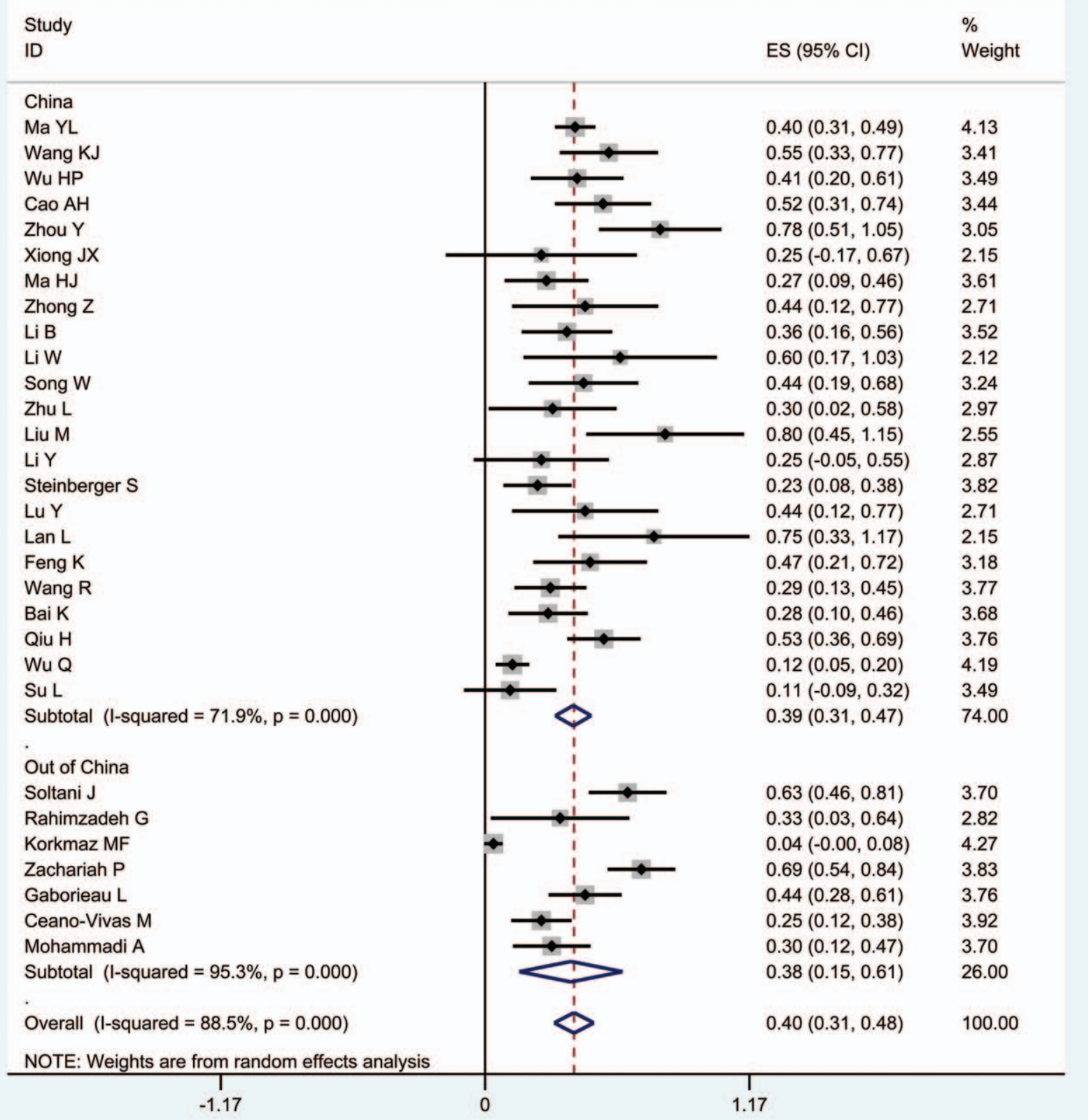

Figure 3. Forest plot of the ground-glass sample. $\mathrm{Cl}=$ confidence interval.

3.3.4. Adjoint sign. Meta-analysis of the random-effect model showed that the prevalence of pleural effusion was $9.1 \%(95 \%$ CI: $2.5 \%-15.7 \%)$ ). Two articles in this study reported cases of white lung ${ }^{[10,16]}$ and 1 article reported a case of pulmonary bullae. ${ }^{[15]}$ A study outside China reported 2 cases of pneumothorax. ${ }^{[37]}$

3.3.5. Subgroup analysis. The heterogeneity of this study was large. To explore the source of heterogeneity, the study was classified according to the region where the study took place (China and non-China) and grouped by the rate of abnormal pulmonary CT findings and ground-glass sample index. The results of each subgroup were consistent with the overall results, and there was no significant difference between the heterogeneity of each subgroup and the whole group, indicating that regional differences were not the main source of heterogeneity (Figs. 2 and 3).

3.3.6. Sensitivity analysis. Sensitivity analysis was carried out for the observation indicators of abnormal changes in CT. After each study was eliminated successively, statistics were recombined, and the results showed no directional change, indicating relatively stable results (Fig. 4).

\subsection{Publication bias}

A funnel plot was drawn for the meta-analysis of abnormal lung CT indicators, and the results showed that the distribution at the left and right of each study point was asymmetrical (Fig. 5), the 


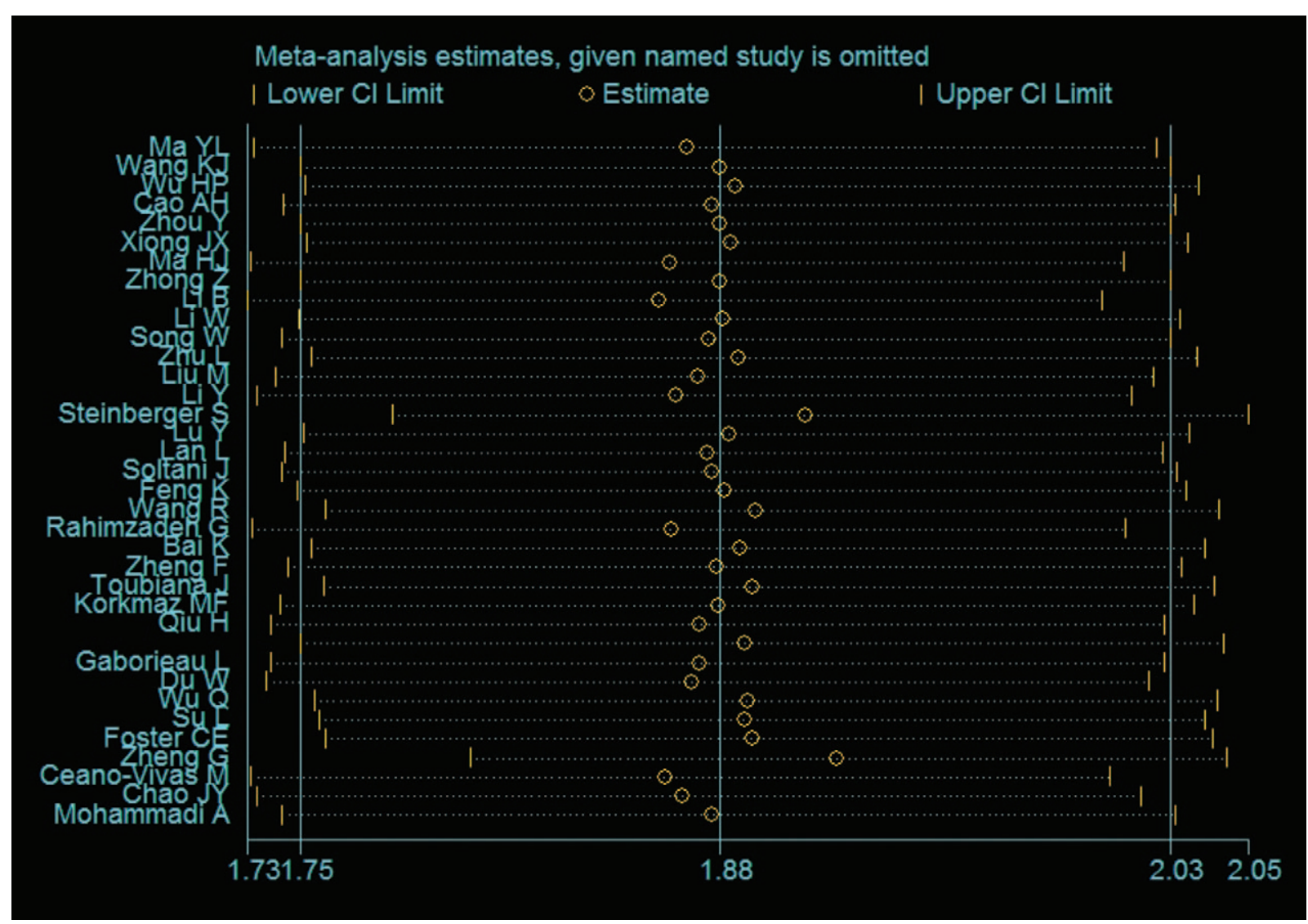

Figure 4. Sensitivity analysis of abnormal CT. Cl = confidence interval, CT = computed tomography.

$P$ values of Egger and Begg tests were .002 and .466 , respectively, indicating that publication bias may exist in this study.

\section{Discussion}

Chest CT examination is a method used to diagnose COVID-19. CT manifestations of COVID-19 in adults mainly include patchy and segmental ground-glass density shadows in 1 or both lungs, or nodular shadows with surrounding ground-glass density

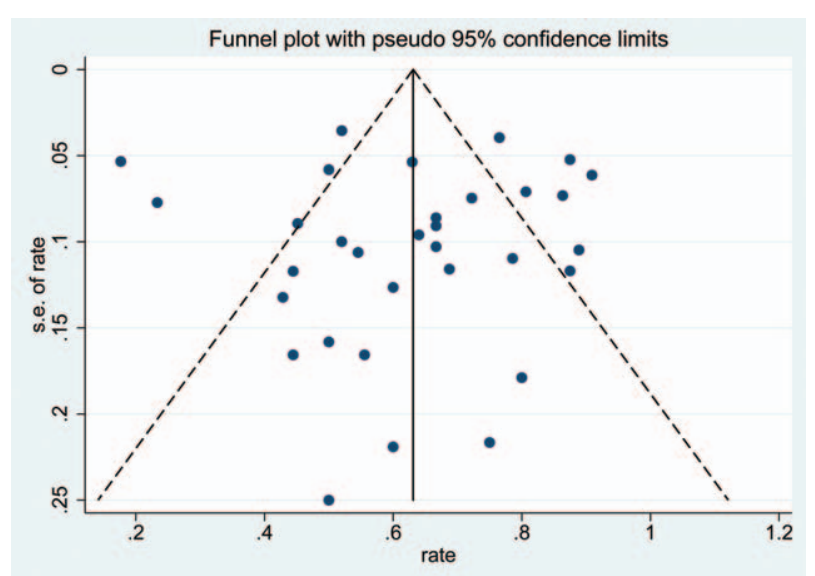

Figure 5. Funnel plot of CT anomaly rate. CT = computed tomography. shadows. It is mainly distributed around the vascular bundle, peripheral dorsal subpleural lung, and the lower lobes of both lungs, with the long axis parallel to the pleura. Air bronchogram and paving stone signs, among others, can also be observed. ${ }^{[4]}$ In a systemic review, including 4121 cases of adult COVID-19 patients, it was found that the prevalence of typical ground-glass opacity was $68.1 \%(95 \%$ CI: $56.9 \%-78.2 \%)$ and that most patients had bilateral lung involvement $(73.8 \%$; $95 \% \mathrm{CI}$ : $65.9 \%-81.1 \%)$ or lesions in multiple lung lobes $(67.3 \% ; 95 \%$ CI: $54.8 \%-78.7 \%) .{ }^{[48]}$ Compared to adults, the current study found that the detection rate of abnormal CT findings in children was only $63.2 \%$ (95\% CI: $55.8 \%-70.6 \%$ ), which is similar to the study of the European Society of Paediatric Radiology Cardiothoracic Imaging Taskforce (64\%). ${ }^{[4]}$ Furthermore, the subgroup analysis showed that the basal rates of abnormal CT findings in and outside of China were $61 \%$ and $67.8 \%$, respectively. While the incidence of typical ground-glass opacities was $39.5 \%$ (95\% CI: $30.7 \%-48.3 \%$ ), the subgroup analysis revealed that the incidences in and outside of China were $39.2 \%$ and $38.1 \%$, respectively. Kumar et al ${ }^{[50]}$ reported a ground glass shadow incidence of $40 \%$, which was similar to the $39.5 \%$ reported in this study. However, Kumar et al ${ }^{[50]}$ found that $55 \%$ patients had unilateral flash involvement, while this study was mainly affected by bilateral lung $(61.5 \%)$. Generally, the pulmonary CT scans of children do not show typical findings as that of adults.

As for the CT examinations of asymptomatic children, Chinese scholar $\mathrm{Lan}^{[26]}$ found and analyzed 4 cases with CT findings. 
Thin-section CT revealed abnormalities in 3 patients, and 1 patient did not present with any abnormal CT findings. Unilateral lung involvement was observed in 2 patients, and 1 patient showed bilateral lung involvement. In total, 5 small lesions were identified, including ground-glass opacity $(n=4)$ and consolidation $(n=1)$. All lesions had ill-defined margins with peripheral distribution and a predilection for the lower lobes. At present, there is no large-scale study on the differences of lung CT findings between asymptomatic and symptomatic children.

Generally, the symptoms of children are relatively mild. In the current study, 2 cases of white lung were reported from Wuhan Children's Hospital; 1 article reported a case of pulmonary bullae. ${ }^{[14]}$ In addition, a study outside China reported 2 cases of pneumothorax. ${ }^{[37]}$ The symptoms of children are milder than those of adults, which may be related to differences in the angiotensin-converting enzyme 2 (ACE2) receptor. ACE2 is an important binding receptor for SARS-CoV-2. ${ }^{[5,10]}$ The ACE2 receptor is still not quite mature in children and it also has reduced function than that in adults, making them less susceptible to SARS-CoV-2 binding. This also leads to a reduced SARS-CoV2 load. In addition, the immune system of children is still in the phase of development; therefore, the intensity of the immune response (cytokine storm) is not as strong as that in adults, which reduces the damage to the body. ${ }^{[51]}$

Several limitations of our study need to be noted: Studies on children with COVID-19 are rare; there are 10 studies with less than 10 participants. Thus, the inspection efficiency may be insufficient. Most included studies were single-center studies; so, there may have been admission and selection biases. Most included studies were retrospective studies, which could not control for confounding factors. Reference, ${ }^{[10,21,37-38,44-46]}$ the severity of the disease is inconsistent, which may lead to clinical heterogeneity. Last, as the pandemic spreads across the globe, additional data have become available for other regions not well represented in this study; therefore, more updated review and meta-analysis providing data for more regions of the world are needed. All these factors will affect the accuracy of the metaanalysis.

In conclusion, chest CT findings of children with COVID-19 are usually normal or slightly atypical; thus, the CT findings show low sensitivity and specificity. Children diagnosed with COVID-19 are mainly diagnosed through reverse-transcription polymerase chain reaction. For children with a high suspicion of COVID-19, imaging examination shows no abnormalities and conclusions should be drawn cautiously.

\section{Author contributions}

Ji-gan Wang and Yu-fang Mo conceived and designed the study. Ji-gan Wang, Yu-heng Su, Li-chuan Wang, and Qian-qiu Qin searched the literature and extracted data. Guang-bing Liu and Meng-Li performed statistical analyses. All authors wrote and reviewed the manuscript.

Conceptualization: Ji-Gan Wang.

Data curation: Ji-Gan Wang, Yu-Fang Mo, Meng Li.

Formal analysis: Ji-Gan Wang.

Methodology: Yu-Fang Mo, Guang-bing Liu.

Software: Yu-heng Su.

Validation: Yu-heng Su.

Visualization: Li-chuan Wang, Qian-qiu Qin.

Writing - original draft: Ji-Gan Wang, Yu-heng Su, Meng Li, Qian-qiu Qin.
Writing - review \& editing: Ji-Gan Wang, Yu-heng Su, Li-chuan Wang.

\section{References}

[1] Li Q, Guan X, Wu P, et al. Early transmission dynamics in Wuhan, China, of novel coronavirus-infected pneumonia. N Engl J Med 2020;382:1199-207.

[2] Remuzzi A, Remuzzi G. COVID-19 and Italy: what next? Lancet 2020;2:10-3.

[3] Cucinotta D, Vanelli M. WHO declares COVID-19 a pandemic. Acta Biomed 2020;91:157-60.

[4] Chan JF, Yuan S, Kok KH, et al. A familial cluster of pneumonia associated with the 2019 novel coronavirus indicating person-to-person transmission: a study of a family cluster. Lancet 2020;395:514-23.

[5] Liu H, Liu F, Li J, Zhang T, Wang D, Lan W. Clinical and CT imaging features of the COVID-19 pneumonia: focus on pregnant women and children. J Infect 2020;80:e7-13.

[6] Ai T, Yang Z, Hou H, et al. Correlation of chest CT and RT-PCR testing for coronavirus disease 2019 (COVID-19) in China: a report of 1014 cases. Radiology 2020;296:E32-40.

[7] Duan YN, Zhu YQ, Tang LL, Qin J. CT features of novel coronavirus pneumonia (COVID-19) in children. Eur Radiol 2020;30:4427-33.

[8] Dong Y, Mo X, Hu Y, et al. Epidemiology of COVID-19 among children in China. Pediatrics 2020;145:e20200702.

[9] Abraham C, Kelly MP, West R, Michie S. The UK National Institute for Health and Clinical Excellence public health guidance on behaviour change: a brief introduction. Psychol Health Med 2009;14:1-8.

[10] Ma YL, Xia SY, Wang M, et al. Analysis of clinical characteristics of 115 children infected by novel coronavirus. Chin J Contemp Pediatr 2020;22:290-3.

[11] Wang KJ, Xu L, Yin H, et al. Clinical features and CT imaging of children with covid-19. J Hubei Univ Med 2020;39:134-8.

[12] Wu HP, Li HF, Chen X, et al. Clinical analysis of covid-19 in 23 children under 18 years of age in Jiangxi province. Chin J Contemp Pediatr 2020;22:419-24.

[13] Cao AH, Duan CH, Qiu BP, et al. Epidemiology and clinical characteristics of children infected with virus from novel coronavirus in Shandong Province. J Shandong Univ (Health Sci) 2020;58:34-40.

[14] Zhou Y, Yang GD, Feng K, et al. Clinical characteristics and chest CT manifestations of covid-19 in infants and young children. Chin J Contemp Pediatr 2020;22:215-20.

[15] Xiong JX, Zhou CY, Zeng WB. CT findings of children with COVID-19. Chongqing Med 2020;49:2837-8.

[16] Ma HJ, Shao JB, Wang YJ, et al. High resolution CT findings of covid-19 children. Chin J Radiol 2020;310-3.

[17] Zhong Z, Xie XZ, Huang W, et al. Chest CT findings and clinical characteristics of children with covid-19 virus. J Central South Univ (Med Sci) 2020;45:236-42.

[18] Li B, Shen J, Li L, Yu C. Radiographic and clinical features of children with coronavirus disease (COVID-19) pneumonia. Indian Pediatr 2020;57:423-6.

[19] Li W, Cui H, Li K, Fang Y, Li S. Chest computed tomography in children with COVID-19 respiratory infection. Pediatr Radiol 2020; 50:796-9.

[20] Song W, Li J, Zou N, Guan W, Pan J, Xu W. Clinical features of pediatric patients with coronavirus disease (COVID-19). J Clin Virol 2020; 127:104377.

[21] Zhu L, Wang J, Huang R, et al. Clinical characteristics of a case series of children with coronavirus disease 2019. Pediatr Pulmonol 2020;55: $1430-2$.

[22] Liu M, Song Z, Xiao K. High-resolution computed tomography manifestations of 5 pediatric patients with 2019 novel coronavirus. J Comput Assist Tomogr 2020;44:311-3.

[23] Li Y, Cao J, Zhang X, Liu G, Wu X, Wu B. Chest CT imaging characteristics of COVID-19 pneumonia in preschool children: a retrospective study. BMC Pediatr 2020;20:227.

[24] Steinberger S, Lin B, Bernheim A, et al. CT features of coronavirus disease (COVID-19) in 30 pediatric patients. Am J Roentgenol 2020;215:1-9.

[25] Lu Y, Wen H, Rong D, Zhou Z, Liu H. Clinical characteristics and radiological features of children infected with the 2019 novel coronavirus. Clin Radiol 2020;75:520-5.

[26] Lan L, Xu D, Xia C, Wang S, Yu M, Xu H. Early CT findings of coronavirus disease 2019 (COVID-19) in asymptomatic children: a single-center experience. Korean J Radiol 2020;21:919-24. 
[27] Soltani J, Sedighi I, Shalchi Z, Sami G, Moradveisi B, Nahidi S. Pediatric coronavirus disease 2019 (COVID-19): an insight from west of Iran. North Clin Istanb 2020;7:284-91.

[28] Feng K, Yun YH, Wang LF, et al. CT image characteristics of children novel coronavirus 2019 infected with 15 cases. Chin J Pediatr 2020; 58:275-8.

[29] Wang R, Ju XL, Xie F, et al. Clinical analysis of 31 cases with novel coronavirus 2019 infection in 6 provinces (autonomous regions) in north China. Chin J Pediatr 2020;58:269-74.

[30] Rahimzadeh G, Ekrami Noghabi M, Elyaderani FK, et al. COVID-19 infection in Iranian children: a case series of 9 patients. J Pediatr Rev 2020;8:139-44.

[31] Bai K, Liu W, Liu C, et al. Clinical analysis of 25 COVID-19 infections in children. Pediatr Infect Dis J 2020;39:e100-3.

[32] Zheng F, Liao C, Fan QH, et al. Clinical characteristics of children with coronavirus disease 2019 in Hubei, China. Curr Med Sci 2020;40:275-80.

[33] Toubiana J, Poirault C, Corsia A, et al. Kawasaki-like multisystem inflammatory syndrome in children during the covid-19 pandemic in Paris, France: prospective observational study. BMJ 2020;369:m2094.

[34] Korkmaz MF, Türe E, Dorum BA, Kılıç ZB. The epidemiological and clinical characteristics of 81 children with COVID-19 in a pandemic hospital in Turkey: an observational cohort study. J Korean Med Sci 2020;35:e236.

[35] Qiu H, Wu J, Hong L, Luo Y, Song Q, Chen D. Clinical and epidemiological features of 36 children with coronavirus disease 2019 (COVID-19) in Zhejiang, China: an observational cohort study. Lancet Infect Dis 2020;20:689-96.

[36] Götzinger F, Santiago-García B, Noguera-Julián A, et al. COVID-19 in children and adolescents in Europe: a multinational, multicentre cohort study. Lancet Child Adolesc Health 2020;4:653-61.

[37] Zachariah P, Johnson CL, Halabi KC, et al. Epidemiology, clinical features, and disease severity in patients with coronavirus disease 2019 (COVID-19) in a children's hospital in New York City, New York. JAMA Pediatr 2020;174:e202430.

[38] Gaborieau L, Delestrain C, Bensaid P, et al. Epidemiology and clinical presentation of children hospitalized with SARS-CoV-2 infection in suburbs of Paris. J Clin Med 2020;9:E2227.

[39] Du W, Yu J, Wang H, et al. Clinical characteristics of COVID-19 in children compared with adults in Shandong Province, China. Infection 2020;48:445-52.
[40] Wu Q, Xing Y, Shi L, et al. Coinfection and other clinical characteristics of COVID-19 in children. Pediatrics 2020;146:e20200961.

[41] Su L, Ma X, Yu H, et al. The different clinical characteristics of corona virus disease cases between children and their families in China - the character of children with COVID-19. Emerg Microbes Infect 2020; 9:707-13.

[42] Foster CE, Moulton EA, Munoz FM, et al. Coronavirus disease 2019 in children cared for at Texas Children's Hospital: initial clinical characteristics and outcomes. J Pediatric Infect Dis Soc 2020;9: 373-7.

[43] Zheng G, Wang B, Zhang H, et al. Clinical characteristics of acute respiratory syndrome with SARS-CoV-2 infection in children in South China. Pediatr Pulmonol 2020;55:2419-26.

[44] de Ceano-Vivas M, Martín-Espín I, Del Rosal T, et al. SARS-CoV-2 infection in ambulatory and hospitalised Spanish children. Arch Dis Child 2020;105:808-9.

[45] Chao JY, Derespina KR, Herold BC, et al. Clinical characteristics and outcomes of hospitalized and critically ill children and adolescents with coronavirus disease 2019 at a tertiary care medical center in New York City. J Pediatr 2020;223:14-9.e2.

[46] Mohammadi A, Mohebbi I, Khademvatani K, et al. Clinical and radiological characteristics of pediatric patients with COVID-19: focus on imaging findings. Jpn J Radiol 2020;38:987-92.

[47] Radiological diagnosis of COVID-19: expert recommendation of the Radiological Society of the Chinese Medical Association (first edition). Chin J Radiology 2020;4:279-85.

[48] Zhu J, Zhong Z, Li H, et al. CT imaging features of 4121 patients with COVID-19: a meta-analysis. J Med Virol 2020;92:891-902.

[49] Shelmerdine SC, Lovrenski J, Caro-Domínguez P, Toso S. Collaborators of the European Society of Paediatric Radiology Cardiothoracic Imaging TaskforceCoronavirus disease 2019 (COVID-19) in children: a systematic review of imaging findings. Pediatr Radiol 2020;50: 1217-30.

[50] Kumar J, Meena J, Yadav A, Yadav J. Radiological findings of COVID19 in children: a systematic review and meta-analysis. J Trop Pediatr 2020;67: doi: 10.1093/tropej/fmaa045.

[51] South AM, Brady TM, Flynn JT. ACE2 (angiotensin-converting enzyme 2), COVID-19, and ACE inhibitor and Ang II (angiotensin II) receptor blocker use during the pandemic: the pediatric perspective. Hypertension 2020;76:16-22. 